\title{
Modeling the Impact of Space Suit Components and Anthropometry on the Center of Mass of a Seated Crewmember
}

\author{
Blackledge, Christopher \\ MEI Technologies \\ Margerum, Sarah \\ Lockheed Martin \\ Ferrer, Mike \\ MEI Technologies \\ Morency, Richard \\ National Aeronautics and Space Administration \\ Rajulu, Sudhakar \\ National Aeronautics and Space Administration
}

\section{ABSTRACT}

The Crew Impact Attenuation System (CIAS) is the energy-absorbing strut concept that dampens Orion Crew Exploration Vehicle (CEV) landing loads to levels sustainable by the crew. Significant COM variations across suited crew configurations would amplify the inertial effects of the pallet and potentially create unacceptable crew loading during launch and landing. The objective of this study was to obtain data needed for dynamic simulation models by quantifying the effects 
of posture, suit components, and the expected range of anthropometry on the COM of a seated individual.

Several elements are required for the COM calculation of a suited human in a seated position: anthropometry, body segment mass, suit component mass, suit component location relative to the body, and joint angles defining the seated posture. Three-dimensional (3D) human body models, suit mass data, and vector calculus were utilized to compute the COM positions for 12 boundary manikins in two different seated postures.

The analysis focused on two objectives: (1) quantify how much the wholebody COM varied from the smallest to largest subject and (2) quantify the effects of the suit components on the overall COM in each seat configuration. The location of the anterior-posterior COM varied across all boundary manikins by about $7 \mathrm{~cm}$, and the vertical COM varied by approximately 9 to $10 \mathrm{~cm}$. The mediolateral COM varied by $1.2 \mathrm{~cm}$ from the midline sagittal plane for both seat configurations. The suit components caused an anterior shift of the total COM by approximately $2 \mathrm{~cm}$ and a shift to the right along the mediolateral axis of $0.4 \mathrm{~cm}$ for both seat configurations. When the seat configuration was in the standard posture the suited vertical COM shifted inferiorly by as much as $1 \mathrm{~cm}$, whereas in the CEV posture the vertical COM had no appreciable change. These general differences were due to the high proportion of suit mass located in the boots and lower legs and their corresponding distance from the body COM, as well as to the prevalence of suit components on the right side of the body.

\section{Keywords:}

Center of mass, COM, space suit, anthropometry, boundary manikins

\section{INTRODUCTION}

This paper describes a method by which three-dimensional coordinates for the center of mass (COM) of a seated human where calculated in unsuited and suited conditions. Designers for the Crew Exploration Vehicle (CEV) requested suited human mass properties to perform their analysis of the Crew Impact Attenuation System (CIAS). The CIAS pallet is the current method by which the entire seated crew would be safely supported during launch and landing operations. This COM data was meant to serve as an input for dynamic modeling of the Orion seat pallet stroking mechanism. The Human Systems Integration Standards (HSIR) document (NASA, CxP 70024, 2009) and the Man-Systems Integration Standards NASA-STD 3000 (MSIS) (NASA, 1995) the precursor to HSIR, define the requirements for human spaceflight. However, suited information does not exist in either document for the current suit architecture or posture required for the CIAS dynamic modeling. As part of a previous project in the $\mathrm{ABF}$, a selection of three-dimensional boundary manikin were developed that represent the critical anthropometric dimension extremes found in the HSIR database (Young, Margerum, Barr, Ferrer, Rajulu, 2008 [A], [B]). The critical dimensions for 6 of the male manikins and 6 of the female 


\section{[Type text]}

manikins were used in this analysis for calculation of whole body COM. The suit information used to calculate the mass properties was taken from data provided by the Extravehicular Activity Project Office on the most recent Cx Launch, Entry, and Abort (LEA) configuration suit (NASA, CxE-EM-2009-0001, 2009).

\section{METHODOLOGY}

The general approach used in this paper to calculate the human mass properties was to utilize PolyWorks ${ }^{\circledR}$ software to segment the boundary manikin scans and determine the center of volume (COV) of body segments, apply a density function to determine the COM, and then a custom MATLAB $®$ script then rotated the COM positions into the seated configuration. The composite COM position was then calculated in 3-D space. Suit components were treated as point masses and were positioned along the body segment based on that segment's length and COM.

\section{ASSUMPTIONS}

Several simplifying assumptions are required for the calculation of mass properties for human data. Without these assumptions the mass properties would have to be empirically measured using methods exceeding the resource limitations of the current project. The assumptions applicable to the data reported are as follows: 1) the human body is modeled as a rigid object composed of linked segments; no soft tissue deformation, spinal curvature, or movement of internal masses are accounted for. 2) The density of the human body is assumed to be a homogeneous 1000 $\mathrm{kg} / \mathrm{m}^{\wedge} 3$. This value was taken from similar human mass studies (Chandler, et al., 1975; Young, et al.,1983) and is applicable within the range of suited pressures defined in HSIR (NASA, CxP 70024, 2009). 3) It is also assumed that the body segment planes are close to joint centers of rotation.

\section{AXIAL SYSTEM}

The axial coordinate system used for all data presented in this paper has its origin at the whole-body COM and is related to the typical body planes of symmetry. The positive $\mathrm{x}$-direction is described as extending in the anterior direction out of the chest in the sagittal plane and perpendicular to the frontal plane. The y-direction extends laterally out of the left side of the body in the frontal plane and perpendicular to the sagittal plane. The positive z-direction is described as extending superiorly out of the top of the head in the frontal plane and perpendicular to the transverse plane. The axial directions can be seen in Figure 1. 


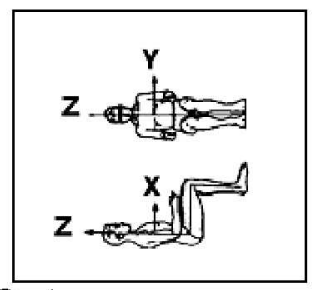

Figure 1: Example Seated Axial System

\section{CEV SEATED POSTURE}

The assumed seated posture for the current CEV configuration was a variation on the standard seated posture described in MSIS (Figure 2), hereby called the MSIS posture. The axial system was anatomically based and COM locations were referenced to the seat pan and seat back, thus independent of seat positioning (i.e. recumbent versus upright). Because the seat configuration impacts the leg posture, the joint angles used to describe the leg seated posture were taken from a previous ABF evaluation of the Orion CEV mockup. A list of the joint angles and associated anatomical landmarks used for the CEV seat configuration are provided in Table 1.

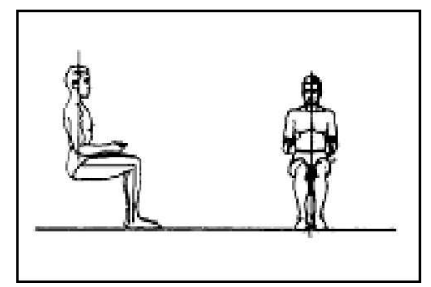

Figure 2: MSIS Standard Seated Position

Table 1: Calculated CEV Seated Configuration Joint Angles

\begin{tabular}{ccc}
\hline Joint Angle & Associated Points & Angle Value (degrees) \\
\hline Shoulder & Elbow, Acromion, Side of Torso & 0 \\
Elbow & Acromion, Elbow, Wrist & 90 \\
Hip & Torso, Hip, Knee & 86 \\
Knee & Hip, Knee, Ankle & 75 \\
\hline
\end{tabular}

\section{BODY SEGMENTS}

The method used to divide the body into rotatable segments followed the body segments used in MSIS and HSIR. The body was broken down along joint centers of rotation or anatomical planes into 17 segments. The segmentation divisions are shown in Figure 3. The body scan segmentation was done using the PolyWorks® software to construct bisecting planes at the anatomical reference points. Examples of these planes along with the associated T-pose posture in PolyWorks ${ }^{\circledR}$ are also shown in Figure 3. 


\section{[Type text]}

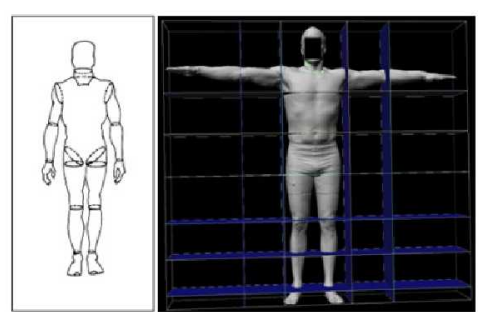

Figure 3: Body Segments and PolyWorks $®$ T-Pose and Segment Planes

\section{DETERMINATION OF SEGMENTAL COM}

After the body scan segmentation was complete the open segment ends were closed to create solid segment volumes. PolyWorks $®$ was used to output the center of volume (COV) coordinates, relative to a body coordinate system centered at the navel, for each of these volumes. As a constant density of the body segments was assumed, the segment COV location coincided with the segment center of mass (COM) location. The resulting segmental COM locations and anatomical body landmarks were exported from PolyWorks $($ for inclusion into a MATLABß program.

\section{SUITED COMPONENTS}

The suited data used for this paper was based on the most current Cx LEA configuration suit information available at the time. Because the suit architecture was still early in development at the time of this paper, an assumption was made that the suit masses were applicable for the entire size range of manikins used, essentially creating a 'one-size fits all' suit. No sizing rings or reduction of materials were accounted for in the application of suited components.

For the center of mass calculation, all suit components were considered a point mass and are applied to either the associated body segment COM; translated along the long axis of the body segment some distance from the COM based on anthropometry; or were positioned in reference to a set of anthropometric points and measures. A diagram illustrating the COM line-of-action translation is shown in Figure 4 below. The percentages for translation distance were determined from conversations with EVA personnel familiar with the suit components and suit fitting. Position vectors in the body centered coordinate system were determined to mathematically apply the suit to the boundary manikins. 


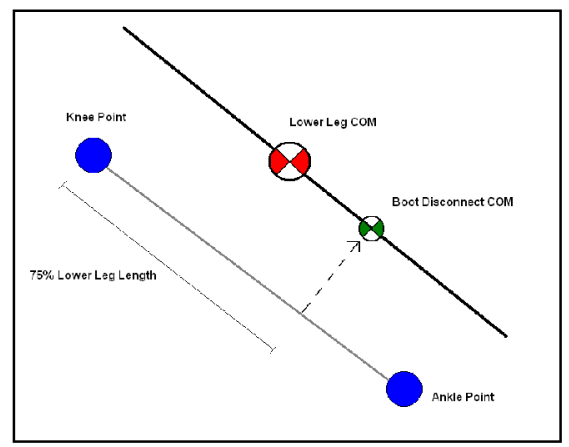

Figure 4: Suit Segment Translation Diagram

Suit components that are mostly uniform in composition and are evenly distributed over the body had their total mass divided by a percentage per body segment, and that percent of component mass was added to the mass of the corresponding body segment. This resulted in no direct change to the body segment's composite COM position.

Rigid suit components such as bearings and disconnects had their point masses positioned in-line with the applicable body segment COM as a function of that segment's anthropometric length, as shown in Figure 4.

The flexible material of the suit located between segment disconnects and bearings, referred to as soft goods, were treated differently than the soft components distributed evenly over the body. Certain soft good components differed in thickness and were segmented differently due to disconnect points and bearings. For these soft good components, a mass per suit segment was provided and positioned in-line with the body segment COM, at the midway point between an anatomical landmark and a bearing or disconnect.

Miscellaneous rigid suit components, except for the helmet, were placed using a similar methodology as the bearing and disconnects. The difference with these components is that they were not placed along the body segment COM. Most of these components were positioned along the body surfaces. Vector calculus and available anatomical reference points were used to determine a displacement position vector for each of these suit components on a case-by-case basis. The helmet and its associated components were assumed to be evenly distributed on the head and were attributed directly to the head's COM.

\section{SEGMENTAL ROTATION}

Once the body was segmented and suit component parts were positioned, all the COM points were rotated from the standing T-pose position into the MSIS seated and CEV seated position. The rotations were accomplished by multiplying the position vectors by a series of rotation matrices hierarchically down the body at anatomical joint rotation center points. 


\section{[Type text]}

\section{WHOLE BODY COM}

The calculation for the whole-body suited COM in the MSIS and CEV seated postures is based off of the general equation for composite centroid calculation in three dimensions as seen in Equation 1 below.

\section{Equation 1: 3D Composite Centroid Equation}

$$
\overrightarrow{C O M}_{\text {Whole_body }}=\frac{\sum\left(\text { mass }_{i} * \overrightarrow{C_{\text {OM }}}\right)}{\sum m a s s_{i}}
$$

This equation was populated with all the body segment COMs and suit segment COMs along with their position vectors to output a position vector describing the composite center of mass in relation to the anatomical origin established in PolyWorks $®$.

Once the whole-body COM position vector was determined (Figure 5), it was set as the global origin for the coordinate system. The resulting segmental COM position vectors and body landmark vectors can then be broken down into axial components for each selected coordinate frame of reference. The COM locations were referenced to an artificial seat pan and seat back to provide a frame of reference for modeling purposes. The unsuited total COM, suited total COM, and body landmarks were then exported as the final results. The unsuited COM results obtained from the method described here were validity checked against the regression equations provided in MSIS (NASA, 1995, p. 3-65) and were analogous to COM displacement value range provided by the MSIS equations.

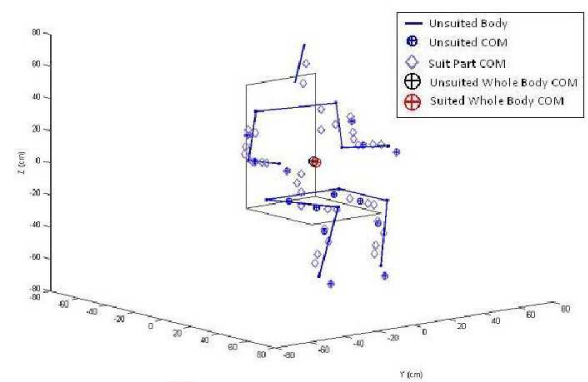

Figure 5: Representative COM Output of a MSIS Suited Seated Individual from MATLAB® 


\section{RESULTS}

\section{MSIS WHOLE BODY COM}

The range of the whole-body center of mass using the MSIS standardized sitting posture (Figure 2) in both the unsuited and suited configurations is provided in Table 2. The data is provided with respect to selected hardware locations, referenced as such in order to reconstruct position and placement using the CAD model of the CIAS.

Table 2: COM range for 12 boundary manikins of a MSIS Suited Individual

\begin{tabular}{lcccccc}
\hline & $\begin{array}{c}\text { Unsuited } \\
\text { Minimum } \\
(\mathrm{cm})\end{array}$ & $\begin{array}{c}\text { Unsuited } \\
\text { Maximum } \\
(\mathrm{cm})\end{array}$ & $\begin{array}{c}\text { Unsuited } \\
\text { Range } \\
(\mathrm{cm})\end{array}$ & $\begin{array}{c}\text { Suited } \\
\text { Minimum } \\
(\mathrm{cm})\end{array}$ & $\begin{array}{c}\text { Suited } \\
\text { Maximum } \\
(\mathrm{cm})\end{array}$ & $\begin{array}{c}\text { Suited } \\
\text { Range } \\
(\mathrm{cm})\end{array}$ \\
\hline Seat Back & 18.0 & 24.9 & 6.9 & 19.8 & 27.0 & 7.2 \\
$\begin{array}{l}\text { Seat Pan } \\
\text { From Hip Midline }\end{array}$ & 24.0 & 32.8 & 8.8 & 23.0 & 32.8 & 9.8 \\
$\begin{array}{l}\text { From Shoulder } \\
\text { Midline }\end{array}$ & -0.3 & 0.5 & 0.8 & -0.5 & 0.9 & 1.4 \\
$\begin{array}{l}\text { From Unsuited } \\
\text { COM as Midline }\end{array}$ & -0.1 & 1.1 & 1.2 & 0.3 & 1.4 & 1.2 \\
\hline
\end{tabular}

1- negative value means the BODY COM is to the left of the midline sagittal plane, positive means the BODY COM is to the right of midline sagittal plane

\section{CEV WHOLE BODY COM}

The range of the whole-body center of mass using the CEV sitting posture (Table 1) in both the unsuited and suited configurations is provided in Table 3 . Identically to the MSIS posture, the data is provided with respect to selected hardware in order to assist in the reconstruction of the COM position and placement within the CAD models.

Table 3: COM range for 12 boundary manikins of a CEV Suited Individual

\begin{tabular}{|c|c|c|c|c|c|c|}
\hline & $\begin{array}{l}\text { Unsuited } \\
\text { Minimum } \\
\text { (cm) }\end{array}$ & $\begin{array}{l}\text { Unsuited } \\
\text { Maximum } \\
\text { (cm) }\end{array}$ & $\begin{array}{l}\text { Unsuited } \\
\text { Range } \\
\text { (cm) }\end{array}$ & $\begin{array}{l}\text { Suited } \\
\text { Minimum } \\
\text { (cm) }\end{array}$ & $\begin{array}{l}\text { Suited } \\
\text { Maximum } \\
(\mathrm{cm})\end{array}$ & $\begin{array}{c}\text { Suited } \\
\text { Range } \\
\text { (cm) }\end{array}$ \\
\hline Seat Back & 17.6 & 24.4 & 6.8 & 19.2 & 26.3 & 7.1 \\
\hline Seat Pan & 24.6 & 33.8 & 9.2 & 23.8 & 34.0 & 10.2 \\
\hline $\begin{array}{l}\text { From Hip Midline } \\
\text { From Shoulder }\end{array}$ & -0.8 & 0.5 & 1.2 & -0.5 & 0.9 & 1.4 \\
\hline $\begin{array}{l}\text { Midline }{ }^{1} \\
\text { From Unsuited }\end{array}$ & -0.1 & 1.1 & 1.2 & 0.3 & 1.4 & 1.2 \\
\hline COM as Midline ${ }^{1}$ & 0.0 & 0.0 & 0.0 & 0.3 & 0.5 & 0.2 \\
\hline
\end{tabular}

1- negative value means the BODY COM is to the left of the midline sagittal plane, positive means the BODY COM is to the right of midline sagittal plane 


\section{[Type text]}

\section{DISCUSSION}

In general, for the MSIS configuration, the suited configuration shifts the COM forward by approximately $2 \mathrm{~cm}$ relative to the seat back with respect to the unsuited configuration. This general change is due to the mass located in the boots and lower legs of the suit and their corresponding distance from the body COM. The suited configuration also shifts the COM down towards the seat pan by up to $1 \mathrm{~cm}$. Again, this is due to the mass located in the boots and lower legs of the suit and their corresponding distance from the body COM. The manikins with longer lower leg lengths experienced the highest amount of change in this regard. Finally, addition of the suit shifts the COM to the right of the midline sagittal plane by approximately $0.4 \mathrm{~cm}$. This shifting of the COM corresponds to the extra components located on the right side of the body, yielding an asymmetry in the suited COM.

The CEV data, in comparison, shifts the suited COM forward by approximately $1.9 \mathrm{~cm}$ relative to the seat back on average. This general change is once again due to the mass located in the boots and lower legs of the suit and their corresponding distance from the body COM. However, this change does not match the MSIS posture because the knees have been drawn closer to the chest from the acute hip angle effects. Unlike the MSIS configuration, the CEV does not have any appreciable change due to the suit for the vertical COM. This is attributable once again to the pulling of the legs toward the chest, i.e. shifting the relative weight vertical. Similar to MSIS, the CEV configuration has the identical shift of the body COM towards the right of the midline sagittal plane due to the extra suit components on the right side.

Other interesting points to note in the data is that there is a variation in the COM locations across the subjects for both the unsuited and suited conditions in each seat posture (Tables 2 and 3): the anterior- posterior COM varies by approximately $7 \mathrm{~cm}$, the vertical COM varies by approximately $9-10 \mathrm{~cm}$, and the right-left COM varies by approximately $1.2 \mathrm{~cm}$ around the midline sagittal plane over the range of subjects. The scale of variation was not anticipated during the initial hypothesis, especially in the anterior-posterior direction.

\section{CONCLUSION}

Based on the variation observed in the results, it is highly recommended that during incorporation of individual crew mass and COM data, that care is exercised in assessing the impact of overall crew mass and COM locations for a crew complement of 2,3 , or 4 during dynamic modeling of the CIAS. Care must be taken in regards to proper set up and validation of the various permutation combinations due to the variation in individual sitting position within the CEV seats, the individual variation of the COM placement relative to the seat, the impacts of the various suit components, as well as the overall group variation in body anthropometry in the CEV. The benefits of this study are twofold, first the 
methodologies with which to predict overall COM for both an unsuited and suited individual in a unique posture underwent a proof of concept and second, the estimated impact of the suit on a seated individual was determined. The results can further be refined as more definitive suit mass components and their associated center of mass locations are developed in the prototype phase of the suit development process. This study is just the preliminary step in assessing the impact of the suited crewmembers on the larger vehicle as a whole.

\section{REFERENCES}

Chandler, R.F., et al. (1975). Investigation of Inertial Properties of the Human Body. Aerospace Medical Research Laboratory, Aerospace Medical Division, Air Force Systems Command, AD-A016485. Wright-Patterson Air Force Base, Ohio.

National Aeronautics and Space Administration (NASA). (2009). Human-Systems Integration Requirements (HSIR), CxP 70024, Revision C. Houston, Texas.

National Aeronautics and Space Administration (NASA). (1995). Man-Systems Integration Standards (MSIS) NASA-STD-3000, Volume II, Revision B. Houston, Texas.

National Aeronautics and Space Administration (NASA). (2009). EVA Suit Segment/Seat Interface Analysis, ODAC-4 Initialization Data, CxE-EM-20090001. Houston, Texas.

Young, J.W., et al. (1983). Anthropometrics and Mass Distribution Characteristics of the Adult Female. FAA Civil Aeromedical Institute, Federal Aviation Administration, AD-A143096. Oklahoma City, Oklahoma.

Young K., Margerum S., Barr A., Ferrer M., Rajulu S. (2008). Generation of Boundary Manikins Anthropometry. International Conference on Environmental Systems. San Francisco, California. [A]

Young K., Margerum S., Barr A., Ferrer M., Rajulu S. (2008). Derivation of Boundary Manikins: A Principal Component Analysis. Digital Human Modeling for Design and Engineering Conference. Pittsburgh, Pennsylvania. [B] 